\title{
BMJ Open Effectiveness of acute geriatric unit care on functional decline and process outcomes among older adults admitted to hospital with acute medical complaints: a protocol for a systematic review
}

To cite: 0'Shaughnessy Í, Robinson $\mathrm{K}, \mathrm{O}$ 'Connor $\mathrm{M}$, et al. Effectiveness of acute geriatric unit care on functional decline and process outcomes among older adults admitted to hospital with acute medical complaints: a protocol for a systematic review. BMJ Open 2021;11:e050524. doi:10.1136/ bmjopen-2021-050524

- Prepublication history and additional supplemental material for this paper are available online. To view these files, please visit the journal online (http://dx.doi.org/10.1136/ bmjopen-2021-050524).

Received 23 February 2021 Accepted 13 October 2021
Check for updates

(C) Author(s) (or their employer(s)) 2021. Re-use permitted under CC BY-NC. No commercial re-use. See rights and permissions. Published by BMJ.

For numbered affiliations see end of article.

Correspondence to Íde 0'Shaughnessy; Ide.0Shaughnessy@ul.ie

\section{ABSTRACT}

Introduction Older adults are clinically heterogeneous and are at increased risk of adverse outcomes during hospitalisation due to the presence of multiple comorbid conditions and reduced homoeostatic reserves. Acute geriatric units (AGUs) are units designed with their own physical location and structure, which provide care to older adults during the acute phase of illness and are underpinned by an interdisciplinary comprehensive geriatric assessment model of care. This review aims to update and synthesise the totality of evidence related to the effectiveness of AGU care on clinical and process outcomes among older adults admitted to hospital with acute medical complaints.

Design Updated systematic review and meta-analysis Methods and analysis MEDLINE, Cumulative Index of Nursing and Allied Health Literature, Controlled Trials in the Cochrane Library and Embase electronic databases will be systematically searched from 2008 to February 2021. Trials with a randomised design that deliver an AGU intervention to older adults admitted to hospital for acute medical complaints will be included. The primary outcome measure will be functional decline at discharge from hospital and at follow-up. Secondary outcomes will include length of stay, cost of index admission, incidence of unscheduled hospital readmission, living at home (the inverse of death or institutionalisation combined; used to describe someone who is in their own home at follow-up), mortality, cognitive function and patient satisfaction with index admission. Title and abstract screening of studies for full-text extraction will be conducted independently by two authors. The Cochrane risk of bias 2 tool will be used to assess the methodological quality of the included trials. The quality of evidence for outcomes reported will be assessed using the Grading of Recommendations Assessment, Development and Evaluations framework. A pooled meta-analysis will be conducted using Review Manager, depending on the uniformity of the data. Ethics and dissemination Formal ethical approval is not required as all data collected will be secondary data and will be analysed anonymously. The authors will present the findings of the review to a patient and public involvement

\section{Strengths and limitations of this study}

- This review will be conducted using a standardised and rigorous methodology.

- To ensure scientific rigour when assessing intervention effects, we have chosen only to include trials with a randomised design.

- Four databases covering the biomedical, nursing and allied health peer-reviewed literature will be searched.

- It is proposed that this review will identify the characteristics and components of comprehensive geriatric assessment within an acute geriatric unit in terms of the intervention, staffing and resources.

- The conclusion of the review will be limited if there is significant heterogeneity across trials, which are identified for inclusion and meta-analyses.

stakeholder panel of older adults that has been established at the Ageing Research Centre in the University of Limerick. This will enable the views and opinions of older adults to be integrated into the discussion section of the paper.

PROSPERO registration number CRD42021237633.

\section{INTRODUCTION}

With population ageing worldwide, the number of older adults attending the emergency department (ED) continues to increase, with evidence of growth in attendances over the past decade beyond that expected from international demographic changes. ${ }^{1}$ It is anticipated that the number of adults aged $\geq 65$ years will increase from 1 billion in 2019 to 1.4 billion by 2030 and further increase to 2.1 billion by $2050 .{ }^{2}$ The ED is often the main portal of entry to unscheduled care for older adults who account for up to $25 \%$ of all attendances. ${ }^{3}$ This disproportionate level of ED use by the 
older population has significant ramifications related to ED overcrowding ${ }^{14}$ and is considered a major patient safety concern as well as being associated with reduced quality of patient care. ${ }^{5}$ Older adults are clinically heterogeneous and often present to the ED and acute care services with a non-specific complaint or with classical frailty syndromes, which are often triggered by a minor stressor event ${ }^{6}$ such as an infection or a complication associated with introduction of a new medication. Their complex medical and psychosocial needs may complicate their ED care ${ }^{78}$ and thus increase their susceptibility to adverse outcomes. Up to $60 \%$ of older adults who present to the ED are admitted for inpatient care as demonstrated in a retrospective cohort study of 550 older adults. ${ }^{9}$

There is broad agreement within the literature that hospital admission poses a significant risk for older adults ${ }^{10}$ and that system wide reform is required to manage the associated deterioration that occurs in vulnerable older adults due to prolonged exposure to such an environment. Loyd et al reported a $30 \%$ (95\% CI $24 \%$ to $33 \%$ ) prevalence rate of hospital-associated disability among older adults in their meta-analysis of 15 longitudinal studies of older adults hospitalised in acute care. ${ }^{11}$ The reasons underlying older adults higher rate of adverse outcomes during hospitalisation are multifaceted; however, the most potent intrinsic factor is the clinical condition of frailty. ${ }^{12}$ The concept of frailty suggests that the accumulation of health deficits is more significant in contributing to vulnerability than specific conditions or physical limitations. ${ }^{14}$ The presence of diminished homoeostatic reserves and multiple comorbid conditions leaves older adults more vulnerable to functional decline and serious sequelae. $^{15}$

Changes to organisational structure and processes are therefore required to better meet the needs of older adults within acute care. ${ }^{16}$ Underpinning all the innovative and evidenced based changes in the way care is delivered is comprehensive geriatric assessment (CGA), which is considered more effective than usual care for frail older adults. ${ }^{17}$ CGA was first coined by Rubenstein $e t$ al and is defined as a "multidimensional, interdisciplinary diagnostic process to determine the medical, psychological and functional capabilities of a frail older person in order to develop a coordinated and integrated plan for treatment and long-term follow-up, ${ }^{18}$ Social and nutritional assessments have recently been recognised as important dimensions of CGA. ${ }^{19}$ CGA is both therapeutic and diagnostic and emphasises improving the quality of life and functional status of frail older adults and at the same time, improving prognosis and outcomes. ${ }^{18}$ Ellis et al conducted a Cochrane review of 29 trials and 13766 participants, which synthesised the evidence on the effectiveness and resource use of CGA for older adults admitted to hospital. ${ }^{17}$ Older adults who receive CGA are more likely to be alive and in their own homes after an emergency admission to hospital and are less likely to be admitted to a nursing home at discharge or at three to 12 months' follow-up. However, the authors were unable to determine whether the results show a difference in effect between discrete specialised wards and mobile multidisciplinary teams across several wards as the analysis was underpowered. Therefore, greater understanding of the the specific impact and organisational forms of CGA delivery in acute services are therefore required to advance the evidence base. Dedicated acute geriatric units (AGU), are units designed with their own physical location and structure, which provide care to older adults during admission to hospital for an acute medical illness including acute exacerbations of chronic diseases. ${ }^{20}$ While variations in the definition exist ${ }^{21}$ all are based on the seminal research on AGUs. ${ }^{22}{ }^{23}$ Furthermore, inclusive of all definitions are specialised interdisciplinary teams who embed geriatric competencies into their practice.

The effectiveness of AGU care for older adults admitted to hospital with acute medical disorders was previously examined in a systematic review and meta-analysis of five RCT, four non-randomised trials and two case-control studies. ${ }^{20}$ The five RCTs dated from 1985 to 2000 and found that admission to an AGU conferred a lower risk of functional decline at discharge and older adults were more likely to be living at home after discharge when compared with conventional care units. However, only two of the five RCTs reported on functional decline at discharge; one RCT presented results at 3-month follow-up, with no differences in the incidence of functional decline reported between groups. Meta-analysis of case fatality either in hospital or at 3-month follow-up did not show any significant differences between groups. Furthermore, authors were unable to draw firm conclusions on length of stay (LoS) between groups due to heterogeneity among trials that reported on this outcome. Further studies on caring for older adults in an AGU have shown lower incidence of adverse outcomes such as delirium ${ }^{24}$ and association with trends of lower LoS and greater cost effectiveness when compared with conventional care units. ${ }^{25-27}$ More recently, the impact of AGU care has focused on healthrelated quality of life (HRQoL) and functional decline after discharge from hospital. ${ }^{28} \mathrm{~A}$ prospective controlled trial where an AGU intervention was provided to 206 frail older adults, aged $\geq 75$ years, in need of acute in-patient treatment when compared with routine care $(n=202)$ found that older adults in the intervention group were less likely to present with decline in HRQoL and in activities of daily living (ADL) at 3-month follow-up.

Our review aims to update and synthesise the totality of recent research evidence related to the effectiveness of AGUs among older adults admitted to hospital with acute medical complaints. We hypothesise that older adults admitted to an AGU experience less functional decline and more favourable process outcomes when compared with conventional care units.

\section{METHODS AND ANALYSIS \\ Study design}

This protocol for a systematic review will be conducted in accordance with the Preferred Reporting of Items for Systematic Reviews and Meta-Analyses (PRISMA) Protocols guidelines. ${ }^{29} \mathrm{~A}$ template will be constructed outlining 
the essential items and components of the protocol as provided in online supplemental file 1 . The systematic review and meta-analysis will comply with the reporting guidance outlined in 27-item PRISMA checklist. ${ }^{30}$ The methodology for the review will be underpinned by the Cochrane handbook for systematic reviews of interventions. ${ }^{31}$ To ensure scientific rigour when assessing intervention effects, we have chosen to only include trials with a randomised design. Randomised controlled trials (RCTs) including cluster trials and quasi-RCTs will also be included in the systematic review.

\section{Search strategy}

Searches were carried out in the following electronic databases-MEDLINE, Cumulative Index of Nursing and Allied Health Literature, Cochrane Central Register of Controlled Trials in the Cochrane Library and Embase. A search string was developed using the keywords in the systematic review and meta-analysis published in $2009 .{ }^{20} \mathrm{MeSH}$ terms and associated keywords covering the topics of older adults, AGU, CGA and RCT were applied to relevant databases outlined above. An example search strategy from MEDLINE in Ebsco are presented in online supplemental file 2. Studies were limited from the year 2008 onwards as this review aims to update a previous meta-analysis. ${ }^{20}$ Trials included in the previous version of the review will be integrated into the new evidence found. The reference lists of all potentially eligible studies will be hand searched for additional papers. Studies in all languages will be included; the authors will seek translation for studies published in languages other than English.

\section{Eligibility criteria}

Trials will be included that meet the following inclusion criteria.

\section{Population}

Older adults ( $>65$ years) admitted to the acute care setting for medical reasons.

\section{Intervention}

In-keeping with the definition used by Baztán $e t$ al in the previous meta-analysis, ${ }^{20}$ the intervention comprises an AGU intervention delivered by interdisciplinary teams during the acute phase of illness to prevent functional decline and related complications in older adults admitted to the acute care setting. The 2017 Cochrane report by Ellis $e t$ al will be used as the reference standard when describing the components of CGA across the included trials. ${ }^{17}$ In their report they outline the following components: clinical leadership, structured assessment, multidisciplinary team meetings, goal setting, involving patients and carers in goal setting, outpatient follow-up, ward environment, adequate time, specialty knowledge, experience, competence and tailoring treatment plans to the individual.

\section{Comparison}

Usual care, other non-AGU interventions such as admission to acute medical wards.

\section{Exclusion criteria}

Trials will be excluded if their population is $<65$ years and if the intervention is aimed at specific medical or surgical complaints or specialty units such as stroke or orthogeriatrics. To ensure we did not include trials that evaluate interventions in the sub-acute phase, exclusions will apply where patients have been transferred from other specialty units such as intensive care to an AGU or admitted to an AGU three or more days after a hospital admission.

\section{Outcome variables}

The primary outcome measure will be functional decline on discharge from hospital and at follow-up. Functional decline will be defined as loss of independence in one or more ADLs compared with the status prior to admission. Various ADL indices such as the Katz Index of Independence in $\mathrm{ADL}^{32}$ and Barthel Index,$^{33}$ which use a numerical scale to measure performance in ADLs will be included in our meta-analysis. Secondary outcomes will include LoS, living at home (the inverse of death or institutionalisation combined; used to describe someone who is in their own home at follow-up), mortality, cost of index admission, incidence of unscheduled hospital readmission, cognitive function and patient satisfaction with the index admission. For the outcome living at home, we will not measure the proportion of patients in receipt of personal assistance and/or services. All secondary outcomes will be recorded at discharge from hospital and at follow-up periods reported in trials.

\section{Study selection and data extraction \\ Screening}

References generated from the search strategy will be exported into Endnote software and duplicates deleted. Two authors (ÍO'S and RG) will independently screen relevant studies by title and abstract for eligibility. Studies that are selected by the reviewers as meeting the inclusion criteria will undergo a full text review. If a disagreement about eligibility arises, both authors will meet to come to a consensus. Where consensus cannot be reached, third and fourth authors will be consulted (KR and MO'C).

\section{Data synthesis and analysis}

Data will be independently extracted from the relevant trials by two reviewers (ÍO'S and RG); data from trials in the previous version of the review will also be extracted. The information compiled will include trial authors, year of publication, study population, sample size, interventions provided, controls provided, outcomes measured and duration of follow-up. Data will be gathered into a preprepared Microsoft Excel document. A pooled metaanalyses will be carried out where the data are homogeneous, which will be determined by the outcomes measured and the time points accessed across the included trials. For the primary outcome of functional decline, we will calculate risk ratios with a $95 \%$ CI to determine the intervention effect. The same approach will be applied for all dichotomous secondary outcome measures. For 
continuous outcomes (length of hospital stay and cost of index admission) we will calculate the intervention effect using mean differences (MD) and 95\% CI where trials all used the same method of measurement. Standardised MD and 95\% CI will be applied where trials used different methods of measurement. The median and IQR will be used in the event that the mean and SD are not reported. ${ }^{34}$ Authors will be contacted where data are not available. Data for the meta-analyses will be analysed using Review Manager V.5.4. (Cochrane Collaboration).

We will explore heterogeneity across the trials by visually inspecting the forest plots and the associated $\mathrm{I}^{2}$ statistics. We will consider $\mathrm{I}^{2}>50 \%$ as significant heterogeneity. If $\mathrm{I}^{2}$ is $\leq 50 \%$ we will apply a fixed-effects method. If $\mathrm{I}^{2}$ is greater than $50 \%$, we will explore the individual trial characteristics to identify potential sources of heterogeneity, using preplanned subgroup analyses. Where there is substantial heterogeneity we will perform a meta-analysis using both fixed-effects and random-effects models and we will present the most conservative outcome. We will conduct separate subgroup analyses after excluding trials that were conducted in the previous version of the review (those published in or before year 2000). ${ }^{20}$

We will conduct a sensitivity analysis to explore the effect of the methodological features on outcomes: (1) randomisation process (data will be reanalysed excluding trials with inadequate or unclear allocation concealment) and (2) bias in the measurement of the outcome (reanalysis of trials without evidence of or with unclear masking of outcome assessor).

\section{Quality assessment}

Trials that meet the inclusion criteria, inclusive of trials in the previous version of the review, will be assessed for risk of bias (RoB) using the Cochrane RoB 2 tool. ${ }^{35}$ Two independent reviewers (ÍO'S and RG) will assess each trial'sRoB under the following domains: randomisation process, deviation from intended intervention, missing outcome data, outcome measurement, selective reporting and the overall risk of bias. If a disagreement about RoB arises, both authors will meet to come to a consensus. Where consensus cannot be reached, third and fourth authors will be consulted (KR and MO'C). The Grading of Recommendations, Assessment, Development and Evaluations framework will be used to assess the quality of evidence for outcomes reported and to summarise data narratively. ${ }^{36}$ Outcomes will be graded at one of four levels of evidence-very low certainty, low certainty, moderate certainty and high certainty. ${ }^{37}$

\section{Patient and public involvement}

The authors will present the findings of the review to a patient and public involvement stakeholder panel of older adults that has been established at the Ageing Research Centre in the University of Limerick https://wwwulie/ hri/themes/public-and-patient-involvement-ppi. The focus of this session will be to discuss the findings with this group so that the discussion section of the paper can integrate the views and opinions of older adults.

\section{DISCUSSION}

This review will update and synthesise the evidence relating to the effectiveness of AGU care on patient and process outcomes for older adults who are admitted to hospital with acute medical complaints. The clinical and social complexity of hospitalised older adults is having an impact on delivery of healthcare services internationally. ${ }^{38}$ CGA and related care is considered a complex intervention $^{39}$; therefore, it is proposed that this review will identify the characteristics and components of CGA within an AGU in terms of the intervention, staff profile and resources. The 10 elements of CGA reported by trialists in the 2017 Cochrane review will be used as a reference standard. ${ }^{17}$ This will have relevance for clinicians and policy-makers and will enable recommendations to be made regarding current and future AGU establishment following evidence-based research. We chose functional decline as our primary outcome as maintaining independence in the performance of ADLs is an important determinant of quality of life for older adults. ${ }^{40}$

By synthesising the evidence surrounding AGU care for older adults with acute medical complaints, there is potential for a reduction in a patient's LoS as is the case in orthogeriatric care. ${ }^{41}$ Reducing LoS can potentially preserve an older adults functional status and thus reduce the risk of increased morbidity and mortality. ${ }^{42}$ Subsequently, this can save hospital bed days and overall reduce hospital costs while enabling older adults to live in their community safely for longer.

\section{Ethics and dissemination}

Formal ethical approval is not required for the review as all data collected will be secondary data and will be analysed anonymously. The findings of this review will be disseminated through publication in a peer-review journal and presented at relevant conferences.

\section{Study status}

Database searches have been completed.

\section{Author affiliations}

${ }^{1}$ School of Allied Health, Faculty of Education and Health Sciences, Ageing Research Centre, Health Research Institute, University of Limerick, Ireland, Limerick, Ireland

${ }^{2}$ Department of Ageing and Therapeutics, University Hospital Limerick, Dooradoyle, Limerick, Ireland

${ }^{3}$ Limerick EM Education Research Training (ALERT), Emergency Department, University Hospital Limerick, Dooradoyle, Limerick, Ireland

${ }^{4}$ Department of Medicine, University Hospital Limerick, Dooradoyle, Limerick, Ireland

${ }^{5}$ Department of Occupational Therapy, University Hospital Limerick, Dooradoyle, Limerick, Ireland

Contributors ÍO'S and RG were major contributors in writing the protocol. KR, MO'C and MC contributed to the planning and conceptualisation of the study design, search strategy, and outcome variables. ÍO'S, RG, KR, MO'C, MC, DR, FS, $\mathrm{LC}$ and $\mathrm{AL}$ participated in the study design and critically appraised and edited the 
manuscript. Í'S is the guarantor of the protocol. All authors read and approved the final manuscript.

Funding The authors have not declared a specific grant for this research from any funding agency in the public, commercial or not-for-profit sectors.

Competing interests None declared.

Patient consent for publication Not applicable.

Provenance and peer review Not commissioned; externally peer reviewed.

Supplemental material This content has been supplied by the author(s). It has not been vetted by BMJ Publishing Group Limited (BMJ) and may not have been peer-reviewed. Any opinions or recommendations discussed are solely those of the author(s) and are not endorsed by BMJ. BMJ disclaims all liability and responsibility arising from any reliance placed on the content. Where the content includes any translated material, BMJ does not warrant the accuracy and reliability of the translations (including but not limited to local regulations, clinical guidelines, terminology, drug names and drug dosages), and is not responsible for any error and/or omissions arising from translation and adaptation or otherwise.

Open access This is an open access article distributed in accordance with the Creative Commons Attribution Non Commercial (CC BY-NC 4.0) license, which permits others to distribute, remix, adapt, build upon this work non-commercially, and license their derivative works on different terms, provided the original work is properly cited, appropriate credit is given, any changes made indicated, and the use is non-commercial. See: http://creativecommons.org/licenses/by-nc/4.0/.

ORCID iDs

Íde 0'Shaughnessy http://orcid.org/0000-0001-9332-8619

Katie Robinson http://orcid.org/0000-0003-1008-9857

\section{REFERENCES}

1 Morley C, Unwin M, Peterson GM, et al. Emergency department crowding: a systematic review of causes, consequences and solutions. PLOS One 2018;13:e0203316.

2 World Health Organisation. Integrated care for older people: guidelines on community-level interventions to manage declines in intrinsic capacity. Geneva: World Health Organization, 2017.

3 Galvin R, Gilleit Y, Wallace E. Adverse outcomes in older adults attending emergency departments: a systematic review and metaanalysis of the identification of seniors at risk (ISAR) screening tool. Age Ageing 2017;46:179-86.

4 Van den Heede K, Van de Voorde C. Interventions to reduce emergency department utilisation: a review of reviews. Health Policy 2016;120:1337-49.

5 Carter EJ, Pouch SM, Larson EL. The relationship between emergency department crowding and patient outcomes: a systematic review. J Nurs Scholarsh 2014;46:106-15.

6 Conroy SP, Turpin S. New horizons: urgent care for older people with frailty. Age Ageing 2016;45:579-86.

7 Elliott A, Hull L, Conroy SP. Frailty identification in the emergency department-a systematic review focussing on feasibility. Age Ageing 2017;46:509-13.

8 Ellis G, Marshall T, Ritchie C. Comprehensive geriatric assessment in the emergency department. Clin Interv Aging 2014;9:2033-43.

9 Kennelly SP, Drumm B, Coughlan T, et al. Characteristics and outcomes of older persons attending the emergency department: a retrospective cohort study. QJM 2014;107:977-87.

10 Gilbert T, Neuburger J, Kraindler J, et al. Development and validation of a hospital frailty risk score focusing on older people in acute care settings using electronic hospital records: an observational study. Lancet 2018;391:1775-82.

11 Loyd C, Markland AD, Zhang Y, et al. Prevalence of hospitalassociated disability in older adults: a meta-analysis. J Am Med Dir Assoc 2020;21:455-61.

12 Hubbard RE, Peel NM, Samanta M, et al. Frailty status at admission to hospital predicts multiple adverse outcomes. Age Ageing 2017;46:801-6.

13 Clegg A, Young J, lliffe S, et al. Frailty in elderly people. Lancet 2013;381:752-62.

14 Vermeiren S, Vella-Azzopardi R, Beckwée D, et al. Frailty and the prediction of negative health outcomes: a meta-analysis. J Am Med Dir Assoc 2016;17:1163.e1-1163.e17.

15 Morley C, Unwin M, Peterson GM, et al. Emergency department crowding: a systematic review of causes, consequences and solutions. PLoS One 2018;13:e0203316.
16 Lafont C, Gérard S, Voisin T, et al. Reducing "iatrogenic disability" in the hospitalized frail elderly. J Nutr Health Aging 2011;15:645-60.

17 Ellis G, Gardner M, Tsiachristas A, et al. Comprehensive geriatric assessment for older adults admitted to hospital. Cochrane Database Syst Rev 2017;9:CD006211.

18 Rubenstein LZ, Stuck AE, Siu AL, et al. Impacts of geriatric evaluation and management programs on defined outcomes: overview of the evidence. J Am Geriatr Soc 1991;39:8S-16.

19 Conroy SP, Bardsley M, Smith P, et al. Comprehensive geriatric assessment for frail older people in acute hospitals: the how-CGA mixed-methods study. Health Serv Deliv Res 2019;7:1-174.

20 Baztán JJ, Suárez-García FM, López-Arrieta J, et al. Effectiveness of acute geriatric units on functional decline, living at home, and case fatality among older patients admitted to hospital for acute medical disorders: meta-analysis. BMJ 2009;338:b50.

21 Palmer RM, Landefeld CS, Kresevic D, et al. A medical unit for the acute care of the elderly. J Am Geriatr Soc 1994;42:545-52.

22 Calkins E, Naughton BJ. Care of older people in the hospital. In: Calkins E, Boult C, Wagner EH, et al, eds. New ways to care for older people: building systems based on evidence. New York: Springer, 1998: 99-111.

23 Ellis G, Langhorne P. Comprehensive geriatric assessment for older hospital patients. Br Med Bull 2004;71:45-59.

24 Vidán MT, Sánchez E, Alonso M, et al. An intervention integrated into daily clinical practice reduces the incidence of delirium during hospitalization in elderly patients. J Am Geriatr Soc 2009;57:2029-36.

25 Borenstein JE, Aronow HU, Bolton LB, et al. Identification and teambased interprofessional management of hospitalized vulnerable older adults. Nurs Outlook 2016;64:137-45.

26 Barnes DE, Palmer RM, Kresevic DM, et al. Acute care for elders units produced shorter hospital stays at lower cost while maintaining patients' functional status. Health Aff 2012;31:1227-36.

27 Zelada MA, Salinas R, Baztán JJ. Reduction of functional deterioration during hospitalization in an acute geriatric unit. Arch Gerontol Geriatr 2009;48:35-9.

28 Ekerstad N, Karlson BW, Dahlin Ivanoff S, et al. Is the acute care of frail elderly patients in a comprehensive geriatric assessment unit superior to conventional acute medical care? Clin Interv Aging 2017;12:1-9.

29 Shamseer L, Moher D, Clarke M, et al. Preferred reporting items for systematic review and meta-analysis protocols (PRISMA-P) 2015: elaboration and explanation. BMJ 2015;349:g7647.

30 Page MJ, McKenzie JE, Bossuyt PM, et al. The PRISMA 2020 statement: an updated guideline for reporting systematic reviews. BMJ 2021;372:n71.

31 Higgins JPT, Thomas J, Chandler J. Cochrane handbook for systematic reviews of interventions version 6.1, 2020.

32 Katz S, Ford AB, Moskowitz RW, et al. Studies of illness in the aged. the index of ADL: a standardized measure of biological and psychosocial function. JAMA 1963;185:914-9.

33 Mahoney FI, Barthel DW. Functional evaluation: the barthel index. Md State Med J 1965;14:61-5.

34 Wan X, Wang W, Liu J, et al. Estimating the sample mean and standard deviation from the sample size, median, range and/or interquartile range. BMC Med Res Methodol 2014;14:135.

35 Sterne JAC, Savović J, Page MJ, et al. Rob 2: a revised tool for assessing risk of bias in randomised trials. BMJ 2019;2:14898.

36 Balshem $\mathrm{H}$, Helfand $\mathrm{M}$, Schünemann $\mathrm{HJ}$, et al. Grade guidelines: 3. rating the quality of evidence. J Clin Epidemiol 2011;64:401-6.

37 Murad MH, Mustafa RA, Schünemann HJ, et al. Rating the certainty in evidence in the absence of a single estimate of effect. Evid Based Med 2017;22:85-7.

38 Ruiz M, Bottle A, Long S, et al. Multi-morbidity in hospitalised older patients: who are the complex elderly? PLoS One 2015;10:e0145372.

39 Craig P, Dieppe P, Macintyre S, et al. Developing and evaluating complex interventions: the new medical research council guidance. BMJ 2008;337:a1655.

40 Courtney MD, Edwards HE, Chang AM, et al. Improved functional ability and independence in activities of daily living for older adults at high risk of hospital readmission: a randomized controlled trial. $J$ Eval Clin Pract 2012;18:128-34.

41 González-Montalvo Jl, Alarcón T, Mauleón JL, et al. The orthogeriatric unit for acute patients: a new model of care that improves efficiency in the management of patients with hip fracture. Hip Int 2010;20:229-35.

42 Grimmer K, Luker J, Beaton K, et al. Trialing individualized interventions to prevent functional decline in at-risk older adults (TRIIFL): study protocol for a randomized controlled trial nested in a longitudinal observational study. Trials 2013;14:266. 